\title{
THE SEMICENTER OF AN ENVELOPING ALGEBRA IS FACTORIAL
}

\author{
LIEVEN LE BRUYN ${ }^{1}$ AND ALFONS I. OOMS
}

\begin{abstract}
Let $L$ be a finite-dimensional Lie algebra over a field $k$ of characteristic zero, and $U(L)$ its universal enveloping algebra. We show that the semicenter of $U(L)$ is a UFD. More generally, the same result holds when $k$ is replaced by any factorial ring $R$ of characteristic zero.
\end{abstract}

Introduction. Throughout this note, $L$ will be a nonzero finite-dimensional Lie algebra over a field $k$ of characteristic zero. Let $U(L)$ be the universal enveloping algebra of $L$ with center $Z(U(L))$ and $D(L)$ will be the division ring of quotients of $U(L)$ with center $Z(D(L))$. For each $\lambda \in L^{*}$, we denote by $D(L)_{\lambda}$ the set of those $u \in D(L)$ such that $x u-u x=\lambda(x) u$ for all $x \in L$. Its elements are called the semi-invariants of $D(L)$ relative to $\lambda$. Clearly, $D(L)_{\lambda} \cdot D(L)_{\mu} \subset D(L)_{\lambda+\mu}$ for all $\lambda, \mu \in L^{*}$. We denote by $\Lambda_{D}(L)$ the subgroup of $L^{*}$ consisting of those $\lambda \in L^{*}$ such that $D(L)_{\lambda} \neq 0$. The sum of the $D(L)_{\lambda}$ is direct and is a $\Lambda_{D}(L)$-graded subalgebra $\operatorname{Sz}(D(L))$ of $D(L)$, called the semicenter of $D(L)$. If we put $U(L)_{\lambda}=$ $U(L) \cap D(L)_{\lambda}$, then the sum $\operatorname{Sz}(U(L))$ of the $U(L)_{\lambda}$ is called the semicenter of $U(L)$. It is a commutative subalgebra of $U(L)$, which never reduces to $k$ and which is stable under all automorphisms of $U(L)$.

If $k$ is algebraically closed, C. Moeglin has shown that $\operatorname{Sz}(U(L))$ is a unique factorization domain [11]. The main aim of this note is to extend this result to any base field of characteristic zero. We wish to thank Professor F. Van Oystaeyen for his helpful comments.

1. Factoriality of $\operatorname{Sz}(D(L))$. Using the important result $[8,9,12]$ that each nonzero two-sided ideal of $U(L)$ contains a nonzero semi-invariant, it is fairly easy to check that $w$ is a semi-invariant of $D(L)$ if and only if $w$ is a quotient of two semiinvariants of $U(L)$ [6, Proposition 1.8]. It follows that $\operatorname{Sz}(D(L))$ is the localization of $\mathrm{Sz}(U(L))$ at the multiplicative system of nonzero semi-invariants of $U(L)$.

Proposition 1. The semicenter $\mathrm{Sz}(D(L))$ of $D(L)$ is a factorial domain (UFD).

Proof. Clearly, $\mathrm{Sz}(D(L))$ is graded by the torsion-free abelian (hence totally ordered) group $\Lambda_{D}(L)$. By [3, Corollary 3.4(1)] we have to verify that $\operatorname{Sz}(D(L))$

Received by the editors April 9, 1984.

1980 Mathematics Subject Classification. Primary 17B35; Secondary 13F15.

Kev words and phrases. Finite-dimensional Lie algebra, universal enveloping algebra, semicenter, factorial ring.

${ }^{1}$ Research supported by a NFWO/FNRS grant. 
satisfies the ascending chain condition on principal ideals. So, let $\operatorname{Sz}(D(L)) a_{1} \subset$ $\operatorname{Sz}(D(L)) a_{2} \subset \cdots$ be an ascending chain with $a_{i} \in \operatorname{Sz}(D(L))$ for all $i$. Let $k^{\prime}$ be an algebraic closure of $k$ and put $L^{\prime}=L \otimes k^{\prime}$. Then $\operatorname{Sz}\left(U\left(L^{\prime}\right)\right)$ is factorial [11] and thus also is its localization $\operatorname{Sz}\left(D\left(L^{\prime}\right)\right)$. Therefore, there exists a natural number $N$ such that for all $m \geqslant N$ we have $\operatorname{Sz}\left(D\left(L^{\prime}\right)\right) a_{N}=\operatorname{Sz}\left(D\left(L^{\prime}\right)\right) a_{m}$. Hence, we can find a unit $w \in \mathrm{Sz}\left(D\left(L^{\prime}\right)\right)^{*}$ such that $a_{N}=w \cdot a_{m}$. Now, $\Lambda_{D}\left(L^{\prime}\right)$ being totally ordered, every unit $w$ of $\operatorname{Sz}\left(D\left(L^{\prime}\right)\right)$ is homogenous, i.e. a semi-invariant of $D\left(L^{\prime}\right)$. But $w \in D(L)$, so $w$ is a nonzero semi-invariant of $D(L)$ and thus a unit of $\operatorname{Sz}(D(L))$. Consequently, $\operatorname{Sz}(D(L)) a_{N}=\operatorname{Sz}(D(L)) a_{m}$ for all $m \geqslant N$.

2. Factoriality of the extended semicenter. Let $k^{\prime}$ be an algebraic closure of $k$ and put $L^{\prime}=L \otimes k^{\prime}$. Then $U\left(L^{\prime}\right)=U(L) \otimes k^{\prime}$ and each $\sigma \in \operatorname{Gal}\left(k^{\prime} / k\right)=\operatorname{Aut}_{k}\left(k^{\prime}\right)$ can be extended to a $k$-automorphism, also denoted by $\sigma$, of $U\left(L^{\prime}\right)$ and $D\left(L^{\prime}\right)$. The orbit $\left\{\sigma(u): \sigma \in \operatorname{Gal}\left(k^{\prime} / k\right)\right\}$ of $u$ is finite for every $u \in U\left(L^{\prime}\right)$. Furthermore, $u \in U(L)$ iff $\sigma(u)=u$ for every $\sigma \in \operatorname{Gal}\left(k^{\prime} / k\right)$. If $u \in U\left(L^{\prime}\right)$ is a semi-invariant with weight $\lambda$, then $\sigma(u)$ is a semi-invariant of $U\left(L^{\prime}\right)$ with weight $\sigma \circ \lambda \circ \sigma^{-1}$. Therefore, $\operatorname{Gal}\left(k^{\prime} / k\right)$ acts on $\operatorname{Sz}\left(U\left(L^{\prime}\right)\right)$.

LeMma 2. Let $u, v$ be nonzero elements of $U(L)$. If $u v$ and $v$ belong to $\operatorname{Sz}(U(L))$, then so does $u$.

Proof. By assumption, $v=v_{1}+\cdots+v_{n}$ and $(*) u v=w_{1}+\cdots+w_{s}$, where, $v_{j} \in U(L)_{\mu}$ and $w_{r} \in U(L)_{\gamma_{r}}$ with $\mu_{j}, \gamma_{r} \in L^{*}$. Each $v_{j}$ (resp. $\left.w_{r}\right)$ is also a semiinvariant of $U\left(L^{\prime}\right)$ with weight $\mu_{j}^{\prime}$ (resp. $\left.\gamma_{r}\right)$. Because $u v \in \operatorname{Sz}\left(U\left(L^{\prime}\right)\right)$ and $k^{\prime}$ is algebraically closed, $u \in \operatorname{Sz}\left(U\left(L^{\prime}\right)\right)$ by [11]. So, $u=u_{1}+\cdots+u_{m}$, where $0 \neq u_{i} \in$ $U\left(L^{\prime}\right)_{\lambda}$, with $\lambda_{i} \in\left(L^{\prime}\right)^{*}$. We show that $u \in \operatorname{Sz}(U(L))$ by induction on $m$. This is clear if $m=1$, because then $u$ is a semi-invariant of $U(L)$ as $u \in U(L)$. Now, let $m>1$ and take $\sigma \in \operatorname{Gal}\left(k^{\prime} / k\right)$. Then

$$
u=\sigma(u)=\sigma\left(u_{1}\right)+\cdots+\sigma\left(u_{m}\right)
$$

where $\sigma\left(u_{i}\right)$ is a semi-invariant with weight $\sigma \circ \lambda_{1} \circ \sigma^{-1}$. By the uniqueness of the decomposition of $u$, we see that $\left\{\sigma\left(u_{1}\right), \ldots, \sigma\left(u_{m}\right)\right\}=\left\{u_{1}, \ldots, u_{m}\right\}$ and $\left\{\sigma \circ \lambda_{1} \circ \sigma^{-1}, \ldots, \sigma \circ \lambda_{m} \circ \sigma^{-1}\right\}=\left\{\lambda_{1}, \ldots, \lambda_{m}\right\}$. On the other hand, $u v=\sum u_{i} v_{j}$, where $u_{i} v_{j}$ is a semi-invariant with weight $\lambda_{i}+\mu_{j}^{\prime}$.

Regrouping the terms and comparing with (*) we conclude that $\gamma_{1}^{\prime}=\lambda_{i}+\mu_{j}^{\prime}$ for some $i, j$. Hence, $\lambda_{i}\left|L=\left(\gamma_{1}^{\prime}-\mu_{j}^{\prime}\right)\right| L=\gamma_{1}-\mu_{j} \in L^{*}$. Consequently, $\sigma \circ \lambda_{i} \circ \sigma^{-1}=$ $\lambda_{i}$ since these are two $k^{\prime}$-linear functionals, taking the same value on each $x \in L$. Hence $\sigma\left(u_{i}\right)=u_{i}$ for all $\sigma \in \operatorname{Gal}\left(k^{\prime} / k\right)$ yielding that $u_{i}$ is a semi-invariant of $U(L)$. So, $\left(u-u_{i}\right) v=u v-u_{i} v \in \operatorname{Sz}(U(L))$ whence by induction $u-u_{i} \in \operatorname{Sz}(U(L))$ and therefore finally $u \in \operatorname{Sz}(U(L))$.

Corollary 3. $\mathrm{Sz}(D(L)) \cap U(L)=\mathrm{Sz}(U(L))$.

Proof. Let $0 \neq u \in \operatorname{Sz}(D(L)) \cap U(L)$. Then $u=w_{1}+\cdots+w_{m}$, where each $w_{i}$ is a nonzero semi-invariant of $D(L)$. Now, $w_{i}=u_{i} \cdot v_{i}^{-1}$ for some semi-invariants $u_{i}, v_{i}$ of $U(L)$. We may assume that $v_{1}=\cdots=v_{m}=v$. Then $0 \neq v \in \operatorname{Sz}(U(L))$ and $u v=u_{1}+\cdots+u_{m} \in \mathrm{Sz}(U(L))$. By the previous lemma, $u \in \operatorname{Sz}(U(L))$ which finishes the proof. 
As we noticed above, $\operatorname{Gal}\left(k^{\prime} / k\right)$ acts on $\operatorname{Sz}\left(U\left(L^{\prime}\right)\right)$. Its fixed ring under this action, $\operatorname{Sz}\left(U\left(L^{\prime}\right)\right) \cap U(L)$, will be called the extended semicenter of $U(L)$ and will be denoted by $\operatorname{Esz}(U(L))$.

THEOREM 4 [6]. The extended semicenter $\operatorname{Esz}(U(L))$ is a unique factorization domain.

COROllary 5 [6]. Let $u$ be a nonzero semi-invariant of $U(L)$. Then there exist unique irreducible, pairwise nonassociated, semi-invariants $u_{1}, \ldots, u_{n}$ and natural numbers $m_{i}, a \in k^{*}$ such that $u=a \cdot u_{1}^{m_{1}} \cdots u_{n}^{m_{n}}$. Moreover, each $u_{i}$ is prime in $\mathrm{Sz}(U(L))$.

Proof. Clearly, $u \in \operatorname{Esz}(U(L))$ which is a UFD. So, let $u=a \cdot u_{1}^{m_{1}} \cdots u_{n}^{m_{n}}$ be a unique factorization of $u$ into irreducible factors. As $u$ is a semi-invariant, so are $u_{1}, \ldots, u_{n}$ [6]. Next, suppose $u_{i}$ divides $v w$ in $\operatorname{Sz}(U(L))$, where $v, w$ are nonzero elements of $\operatorname{Sz}(U(L))$. In $\operatorname{Esz}(U(L)), u_{i}$ is prime and divides $v w$. Therefore $u_{i}$ divides $v$ (or $w$ ) in $\operatorname{Esz}(U(L))$, i.e. $v=u_{i} y$ for some nonzero $y \in \operatorname{Esz}(U(L))$. Now $u_{i} y$ and $u_{i}$ belong to $\operatorname{Sz}(U(L))$. By Lemma 2, this implies that $y \in \operatorname{Sz}(U(L))$ and thus $u_{i}$ is prime in $\operatorname{Sz}(U(L))$.

The following shows that it may happen that $\operatorname{Esz}(U(L)) \neq \operatorname{Sz}(U(L))$.

EXAmple 6 [6]. Let $L$ be the Lie algebra over $\mathbf{R}$ with basis $x, y, z$ such that $[x, y]=y+z,[x, z]=-y+z$ and $[y, z]=0$. One verifies that $\operatorname{Sz}(U(L))=$ $\mathbf{R}\left[y^{2}+z^{2}\right]$. In $L \otimes \mathbf{C}$ we consider the basis $x, u_{1}=y+i z, u_{2}=y-i z$. Then $\mathrm{Sz}(U(L \otimes \mathbf{C}))=\mathbf{C}\left[u_{1}, u_{2}\right]$ and $\operatorname{Esz}(U(L))=\mathbf{R}[y, z]$.

3. Factoriality of $\operatorname{Sz}(U(L))$. We are now in a position to prove the main result of this note.

THEOREM 7. The semicenter $\operatorname{Sz}(U(L))$ of $U(L)$ is a unique factorization domain.

Proof. From Corollary 5 we know that $\operatorname{Sz}(U(L))$ is a graded UFD, that is, a graded integral domain such that each nonzero, nonunit homogeneous element is a product of prime elements [1, Definition 4.1]. Moreover, the localization of $\operatorname{Sz}(U(L))$ at the multiplicative system of nonzero homogeneous elements, $Q^{g}(\operatorname{Sz}(U(L)))=$ $\mathrm{Sz}(D(L))$, is a UFD by Proposition 1. Invoking [1, Theorem 4.4], we may conclude that $\mathrm{Sz}(U(L))$ is also a UFD.

4. Factoriality of $\mathrm{Sz}(U(\mathfrak{g})), \mathfrak{g}$ being a Lie algebra over a domain $R$. We shall now take a brief look at a more general situation. Let $R$ be an integral domain of characteristic zero, with quotient field $k$. Let $\mathfrak{g}$ be a Lie algebra over $R$ such that $\mathfrak{g}$ is a free $R$-module of finite rank and put $L=\mathfrak{g} \otimes k$. The semicenter $\operatorname{Sz}(U(\mathrm{~g}))$ of $U(\mathrm{~g})$ is defined analogously. The following is easy to verify (see e.g. [13, Lemma 1, p. 3]).

REMARK 8. Let $u, v$ be nonzero elements of $U(\mathrm{~g})$. If $u v$ belongs to $R$, then so do $u$ and $v$.

THEOREM 9. The semicenter $\operatorname{Sz}(U(\mathfrak{g}))$ is factorial if and only if $R$ is factorial.

Proof. Suppose $R$ is factorial. Then D. Reynaud has shown that $A=\operatorname{Sz}(U(g))$ is factorial $[10 ; 13$, Theorem 10, p. 48$]$ in case $k$ is algebraically closed, the latter 
condition being required only in order for Moeglin's result [11] to be applicable to $L$. Therefore the same proof combined with Theorem 7 yields that $A$ is factorial in general. Conversely, let us assume that $A$ is factorial. By Remark $8, R$ and $A$ have the same units if $v \in R$, then $v$ is irreducible in $R$ if and only if $v$ is irreducible in $A$. Now, take any nonzero element $u \in R$. Let $c=c \cdot v_{1}^{m_{1}} \cdots v_{p}^{m_{p}}, c \in A^{*}$, be its unique decomposition into irreducible factors in the factorial ring $A$. By Remark 8 and the foregoing observations, the decomposition is also the unique factorization of $u$ in terms of irreducible factors in the ring $R$, which is therefore factorial.

\section{REFERENCES}

1. D. D. Anderson and D. F. Anderson, Divisibility properties of graded domains, Canad. J. Math. 34 (1982), 196-215.

2. Divisorial ideals and invertible ideals in a graded integral domain, J. Algebra (to appear).

3. D. F. Anderson, Graded Krull domains, Comm. Algebra 7(1) (1979), 79-106.

4. A. Bouvier, Anneaux de Krull gradués, Université Claude-Bernard, Lyon I, 1981.

5. M. Chamarie, Maximal orders applied to enveloping algebras, Proc. Conf. on Ring Theory (Antwerp, 1980), Lecture Notes in Math., vol. 825, Springer-Verlag, Berlin and New York, 1980, pp. 19-27.

6. L. Delvaux, E. Nauwelaerts and A. I. Ooms, On the semi-center of a universal enveloping algebra, J. Algebra (to appear).

7. J. Dixmier, Enveloping algebras, North-Holland Mathematical Library, Vol. 14, North-Holland, Amsterdam, 1977.

8. V. Ginzburg, On the ideals of $U(\mathrm{~g})$ (to appear).

9. M. P. Malliavin, Ultra produit d'algèbres de Lie, Lecture Notes in Math., vol. 924, Springer-Verlag, Berlin and New York, 1982, pp. 157-166.

10. G. Maury and D. Reynaud, Algèbres enveloppantes de R-algèbres de Lie sur certains anneaux $R$, Comm. Algebra 11(7), (1983), 753-769.

11. C. Moeglin, Factorialité dans le algèbres enveloppantes, C. R. Acad. Sci. Paris Ser. A 282 (1976), 1269-1272.

12. __ Idèux bilatères des algèbres enveloppantes, Bull. Soc. Math. France 108 (1980), 143-186.

13. D. Reynaud, Algèbres enveloppantes de R-algèbres de Lie sur certains anneaux $R$, Thèse de troisième cycle, Université Claude Bernard, Lyon I, 1982.

Department of Mathematics, University of ANTWERP, UiA, 2610 WilRiJK, Belgium

Department of Mathematics, University of Limburg, LUC, 3610 Diepenbeek, Belgium 